\title{
MANEJO DOS RESÍDUOS DE SERVIÇOS DE SAÚDE (RSS) EM ESTABELECIMENTOS MÉDICOS VETERINÁRIOS DE FORTALEZA - CE
}

\author{
RAQuel Russo Silveira CorReia e Gemmelle Oliveira SANTOS \\ Instituto Federal de Educação, Ciência e Tecnologia do Ceará (IFCE) \\ <raquelrusso.2006@gmail.com>.<gemmelle@ifce.edu.br>
}

DOI: $10.21439 /$ conexoes.v13i3.1243

\begin{abstract}
Resumo. O objetivo desse trabalho foi diagnosticar as atuais formas de manejo (acondicionamento, coleta, transporte, tratamento e destino final) dos Resíduos de Serviços de Saúde - RSS gerados em 25 estabelecimentos médicos veterinários de Fortaleza-CE. A pesquisa envolveu a aplicação de um questionário baseado nos requisitos da Resolução ANVISA n 306/04 e CONAMA no 358/05 e abrangeu estabelecimentos encontrados em 10 bairros. Observou-se que nos estabelecimentos estudados são gerados resíduos do Grupo A (biológico), B (químico), D (comum) e E (perfurocortante), não havendo geração de rejeitos radioativos (Grupo C). Nenhum estabelecimento cumpre por completo e corretamente todas as etapas do manejo, mas isso não exclui a existência de boas práticas em alguns locais e para determinadas fases do gerenciamento. Em linhas gerais, os estabelecimentos têm maior rigor no cumprimento das etapas iniciais do manejo (segregação e identificação) e maior dificuldade para cumprir a etapa de armazenamento (abrigos de resíduos fisicamente inadequados). A maior parte dos locais visitados afirmou possuir Plano de Gerenciamento de Resíduos de Serviços de Saúde - PGRSS e Licença Ambiental emitida pela Secretaria de Urbanismo e Meio Ambiente - SEUMA, mas se recusou a mostrar tais documentos, descumprindo as orientações legais. Considerando a ausência de informações e dados sobre esse tema em Fortaleza-CE, os resultados da pesquisa podem contribuir para a divulgação do tema e orientar políticas públicas, inclusive relacionadas com a fiscalização.
\end{abstract}

Palavras-chaves: Resíduos de serviços de saúde, manejo, gerenciamento.

\begin{abstract}
This study is aimed to diagnose the current forms of healthcare waste management (conditioning, collection, transportation, treatment and final disposal) in 25 veterinary facilities in the city of Fortaleza, state of Ceará, Brazil. This survey involved a questionnaire based on the requirements of ANVISA (the Brazilian Sanitary Authority) Resolution 306/04 and CONAMA (the Brazilian Environmental Protection Authority) Resolution 358/05, covering establishments located in 10 different districts within the city. We have found that the surveyed facilities generated Class A (biological), B (chemical), D (common) and E (sharp items) wastes, and did not generate any radioactive waste (Class C). No facility fully and correctly complied with all waste management steps, yet this fact did not preclude the existence of good practices in some sites and for certain waste management stages. In general, establishments are more rigorous in complying with the initial waste management stages (segregation and identification) and are less compliant with the storage stage (physically inadequate waste containers). Most of the visited facilities claimed to have a Healthcare Waste Management Plan and the Environmental License issued by the Department of Urbanism and Environment. However, they refused to show such documents, in violation of the legal guidelines. Considering the lack of information and data on this subject in Fortaleza, these results may contribute to raise awareness on this subject and guide public policy, including supervisory regulations.
\end{abstract}

Keywords: Medical waste; handling, management. 


\section{INTRODUÇÃO}

Os Resíduos de Serviços de Saúde (RSS) constituem um sério problema tanto no âmbito da saúde pública como no ambiental em decorrência de suas características físicas e do seu potencial de contaminação.

Segundo Ramos e et al (2011), os RSS compõem parte importante do total dos Resíduos Sólidos Urbanos (RSU) não pela quantidade gerada, mas pelo potencial risco que afeta à saúde ambiental e coletiva. A Agência Nacional de Vigilância Sanitária (ANVISA), por meio da Resolução no 306/04 (BRASIL, 2004) e o Conselho Nacional do Meio Ambiente (CONAMA), por meio da CONAMA no 358/2005, regulamentaram o gerenciamento dos RSS e atribuíram ao gerador a responsabilidade em providenciar seu adequado manejo via elaboração de um Plano de Gerenciamento.

Segundo a CONAMA no 358/2005, os RSS são aqueles provenientes de qualquer unidade que execute atividades de natureza médico-assistencial humana ou animal, gerados em centros de pesquisa, desenvolvimento ou experimentação na área de farmacologia e saúde, incluindo-se os medicamentos e imunoterápicos vencidos ou deteriorados, como também, os resíduos provenientes de necrotérios, funerárias, serviço de medicina legal e barreiras sanitárias.

Diante dessa diversidade, a Organização PanAmericana de Saúde (OPAS, 1997) coloca que a classificação adequada dos resíduos é que permite seu manejo eficiente, econômico e seguro. Essa classificação envolve cinco grupos: grupo A (infectantes), grupo B (químicos), grupo $\mathrm{C}$ (radioativos), grupo D (comum) e grupo E (perfurocortantes).

No contexto urbano, o grande número de estabelecimentos voltados à saúde humana (postos de saúde, clínicas, hospitais, farmácias etc) concentra a maior parte das atenções dos pesquisadores e do poder público, especialmente das secretarias de vigilância sanitária e de meio ambiente, enquanto clínicas veterinárias, pet shop e similares, que também geram RSS em suas atividades, são pouco fiscalizados.

O mercado de produtos e serviços para animais de estimação é um potencial gerador de RSS. Segundo Afonso, Berdasco e Medeiros T Rejowski (2008) o Brasil representa o segundo maior mercado pet do mundo (com $8 \%$ do comércio mundial), ficando atrás somente dos Estados Unidos (que detêm 30\% desse mercado).

A expansão do mercado pet é resultado também do crescimento da urbanização. Conforme estimativa do CFMV (2013) existem no Brasil aproximadamente 106 milhões de animais de estimação (pets).

No Nordeste brasileiro, o mercado pet cresceu $13 \%$, apesar da crise econômica e da recessão de 2015. A estimativa é que o mercado movimente valores superiores a 15 bilhões de reais por ano, abrangendo $0,4 \%$ do PIB nacional.
O mercado pet (que envolve alimentos, medicamentos, treinamento, brinquedos, acessórios, banho, tosa, consulta, exame, vacinação, tratamento, internação, cirurgia, sepultamento) resulta na geração de RSS e, na mesma medida, deveriam demandar procedimentos corretos de gerenciamento desses resíduos.

O objetivo da pesquisa foi diagnosticar as atuais formas de manejo (acondicionamento, coleta, transporte, tratamento e destino final) dos Resíduos de Serviços de Saúde (RSS) gerados em 25 estabelecimentos médicos veterinários de Fortaleza-CE.

Os resultados da pesquisa podem contribuir para a redução da lacuna no número de estudos e dados sobre o assunto na cidade de Fortaleza-CE e orientar políticas públicas, especialmente as de fiscalização.

\section{METODOLOGIA}

A pesquisa classificou-se como aplicada, exploratória, de campo, quantitativa e com uso do método indutivo.

Inicialmente, foi realizada a busca e leitura de arquivos virtuais e impressos sobre o tema principal da pesquisa: resíduos de serviços de saúde. Assim, foi possível aumentar a compreensão sobre o assunto e o material consultado (artigos, monografias, dissertações, teses, leis, decretos, normas, manuais etc.) permitiu construir uma breve análise sobre a classificação dos RSS e conhecer algumas experiências em estabelecimentos médicos veterinários a partir da literatura.

Em um segundo momento foi realizada a elaboração de um questionário para um pré-teste em campo. Sua aplicação ocorreu com o apoio dos estudantes do $5^{\circ}$ semestre da Graduação em Tecnologia e Gestão Ambiental do Instituto Federal de Educação, Ciência e Tecnologia - IFCE (Campus Fortaleza) como pré-requisito de conclusão de uma disciplina na área de resíduos sólidos. Na ocasião, os estudantes visitaram 32 estabelecimentos veterinários em diversos bairros da cidade. Os resultados do trabalho de campo foram discutidos em sala de aula e permitiram aprimorar o questionário definitivo que viria a ser aplicado pela pesquisadora.

A pesquisadora aplicou o questionário definitivo em 25 estabelecimentos médicos veterinários (clínicas, consultórios, pet shop e similares) encontrados em 10 bairros de Fortaleza-CE: Centro, Monte Castelo, Aldeota, Benfica, Ellery, Parquelândia, Farias Brito, Fátima, Meireles e Pici (Figura 1).

A aplicação dos questionários foi realizada em fevereiro e março de 2016 com o representante legal do estabelecimento. Foram colhidos também alguns depoimentos dos demais funcionários. Nas visitas realizou-se alguns registros fotográficos. Por fim, realizou-se o tratamento das informações e dados, e análise quantitativa mediante nova consulta à literatura. 
ANÁLISE MECÂNICA DE UM SOLO ARENO-ARGILOSO ESTABILIZADO COM CAL PARA FINS DE PAVIMENTAÇÃO

Figura 1: Distribuição dos estabelecimentos visitados por bairro (Fortaleza, 2016)

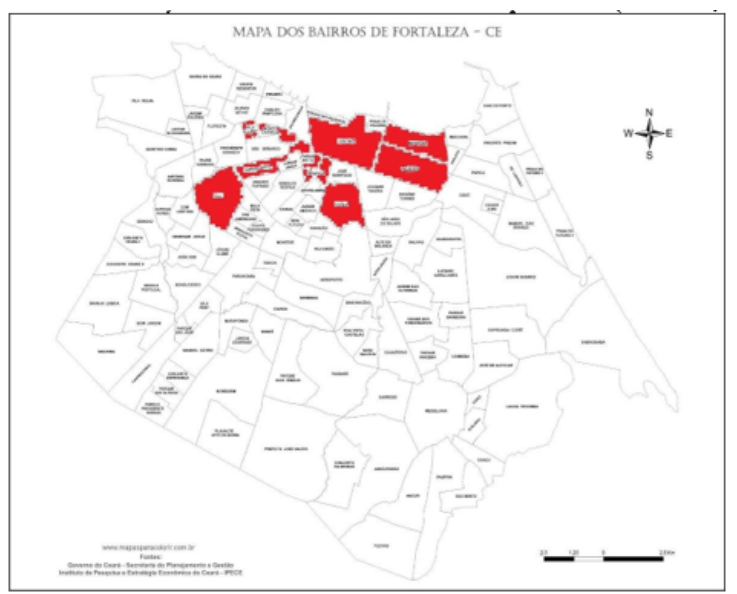

Fonte: Adaptado do site do IPECE.

\section{MATERIAIS E MÉTODOS}

Nos estabelecimentos estudados são gerados resíduos do Grupo A (biológico), B (químico), D (comum) e E (perfurocortante), não havendo geração de rejeitos radioativos (Grupo C). Tais dados se assemelham com os resultados encontrados por Castro et al. (2007) e Pilger e Schenato (2008) em estabelecimentos do mesmo ramo em outras cidades do Brasil.

Conforme Cussiol (2008), ao classificar corretamente os resíduos gerados torna-se possível separá-los conforme sua composição (químicas, físicas ou biológicas), estado da matéria, origem, local de geração e potencial de contaminação.

A segregação possibilita que o gerador realize a manipulação adequada de seus resíduos. Além disso, quando a segregação é feita no momento e local da geração, ela diminui o volume de materiais que precisam de um manejo diferenciado (BRASIL, 2005), o que possibilita a redução de custos no gerenciamento.

A segregação adequada dos resíduos, na visão de Garcia e Zanetti-Ramos (2004), traz benefícios para a comunidade, contribui para a redução da poluição ambiental e implica em menor dano aos recursos naturais.

Os resíduos do Grupo A são aqueles com possível presença de agentes biológicos e por suas características de maior virulência ou concentração podem apresentar risco de infecção. Como se observa (Tabela 1), a maioria dos estabelecimentos geram vacinas $(84 \%)$ e seringas $(80 \%)$ enquanto a minoria gera animais mortos e fetos $(16 \%)$. Cabe destacar que muitos proprietários dos animais falecidos nos consultórios veterinários preferem levar o corpo do animal para providenciar um destino (enterro ou incineração) que deixar sob responsabilidade da clínica.
Tabela 1: Geração de resíduos do Grupo A nos estabelecimentos visitados (Fortaleza, 2016)

\begin{tabular}{l|c}
\hline GRUPO A & \% de estab. \\
\hline $\begin{array}{l}\text { Vacina } \\
\text { (líquido, frasco) }\end{array}$ & 84 \\
\hline Seringa & 80 \\
\hline Algodão com sangue & 68 \\
\hline Gazes com sangue & 56 \\
\hline $\begin{array}{l}\text { Recipientes } \\
\text { contendo fezes, urina e secreções }\end{array}$ & 44 \\
\hline Bolsa de sangue (vencida, sobras) & 40 \\
\hline Resíduos de cirurgia e vísceras & 28 \\
\hline Peças anatômicas (órgãos e tecidos) & 20 \\
\hline Animais mortos e fetos & 16 \\
\hline
\end{tabular}

Fonte: Autores (2016).

Os resíduos do Grupo B (Tabela 2) são aqueles que contêm substâncias químicas que podem apresentar risco à saúde pública ou ao meio ambiente, dependendo de suas características de inflamabilidade, corrosividade, reatividade e toxicidade. Como se observa, a maioria dos estabelecimentos geram resíduos farmacêuticos e medicamentos (96\%) e embalagens de saneantes, desinfetantes $(92 \%)$ enquanto a minoria gera efluentes de processadores de imagem (reveladores e fixadores): 4\%. A recente Política Estadual de Resíduos Sólidos, publicada em 2016, obriga os fabricantes, importadores, distribuidores e comerciantes de medicamentos e outros insumos para saúde a estruturar e implementar sistemas de logística reversa.

Tabela 2: Geração de resíduos do Grupo B nos estabelecimentos visitados (Fortaleza, 2016)

\begin{tabular}{l|c}
\hline GRUPO B & \% de estab. \\
\hline Insumos farmacêuticos e medicamentos & 96 \\
\hline Embalagens de saneantes, desinfetantes & 92 \\
\hline Reagentes para laboratório & 56 \\
\hline Efluentes dos equipamentos de análises clínicas & 12 \\
\hline $\begin{array}{l}\text { Efluentes de } \\
\text { processadores de imagem (reveladores e fixadores) }\end{array}$ & 4 \\
\hline
\end{tabular}
Fonte: Autores (2016).

Os resíduos do Grupo D (Tabela 3) são aqueles que não apresentam risco biológico, químico ou radiológico, podendo ser equiparados aos resíduos domiciliares. Considerando o baixo risco desse grupo de resíduos, alguns estabelecimentos, acertadamente, realizam a coleta seletiva.

Os resíduos do Grupo E (Tabela 4) são materiais perfurocortantes ou escarificantes.

Tão importante quanto segregar os resíduos é realizar corretamente procedimentos de acondicionamento. Na pesquisa realizada, observou-se que $60 \%$ dos estabelecimentos adotam corretamente sacos branco-leitoso para acondicionar os resíduos do Grupo A, mas $28 \%$ dos 
Tabela 3: Geração de resíduos do Grupo D nos estabelecimentos visitados (Fortaleza, 2016)

\begin{tabular}{l|c}
\hline GRUPO D & \% de estab. \\
\hline $\begin{array}{l}\text { Papel de uso } \\
\text { sanitário }\end{array}$ & 100 \\
\hline $\begin{array}{l}\text { Resíduos } \\
\text { provenientes das áreas administrativas }\end{array}$ & 96 \\
\hline $\begin{array}{l}\text { Resíduos de } \\
\text { varrição, flores, podas e jardins }\end{array}$ & 92 \\
\hline Absorventes higiênicos & 88 \\
\hline $\begin{array}{l}\text { Sobras de } \\
\text { alimentos e do preparo de alimentos }\end{array}$ & 84 \\
\hline $\begin{array}{l}\text { Material } \\
\text { utilizado em anti-sepsia }\end{array}$ & 68 \\
\hline $\begin{array}{l}\text { Equipo de } \\
\text { soro e outros similares }\end{array}$ & 32 \\
\hline $\begin{array}{l}\text { Resíduos de Fonte: Autores (2016) } \\
\text { gesso provenientes de assistência à saúde }\end{array}$ & \\
\hline
\end{tabular}

Tabela 4: Geração de resíduos do Grupo E nos estabelecimentos visitados (Fortaleza, 2016)

\begin{tabular}{l|c}
\hline GRUPO E & \% de estab. \\
\hline Agulhas & 84 \\
\hline Escalpes & 80 \\
\hline Ampolas de vidro & 76 \\
\hline Lâminas de barbear & 68 \\
\hline Lâminas de bisturi & 56 \\
\hline Lâminas e lamínulas & 44 \\
\hline Tubos capilares & 32 \\
\hline Micropipetas & 28 \\
\hline Utensílios de vidro quebrados no laboratório & 24 \\
\hline Broca, lima,lanceta & 16 \\
\hline \multicolumn{2}{c}{ Fonte: Autores (2016). }
\end{tabular}

Fonte: Autores (2016).

estabelecimentos usam sacos comuns e de qualquer cor; o que não é permitido, e $12 \%$ não souberam responder, Figura 2

Figura 2: Distribuição dos estabelecimentos visitados por bairro (Fortaleza, 2016)

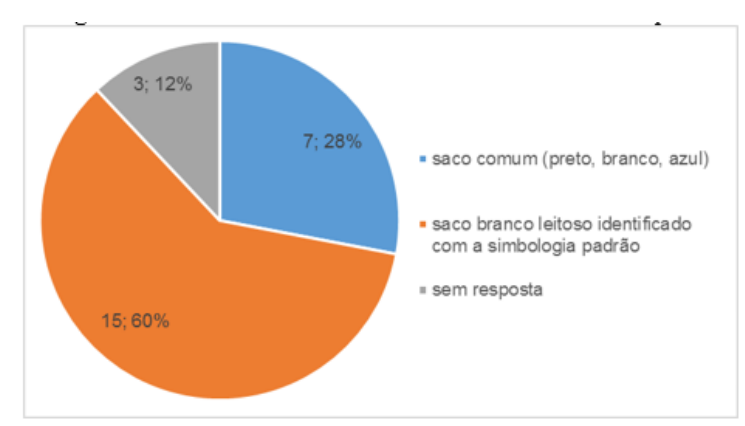

Com relação ao acondicionamento dos resíduos do Grupo B, observou-se que $44 \%$ dos estabelecimentos utilizam recipientes e/ou sacos adequados, 36\% acondicionam erroneamente em saco comum (preto, branco e azul), $16 \%$ mantém o resíduo dentro da própria embalagem do produto e $4 \%$ utiliza frascos com tampa rosqueada e vedante, Figura 3
Figura 3: Distribuição dos estabelecimentos visitados por bairro (Fortaleza, 2016)

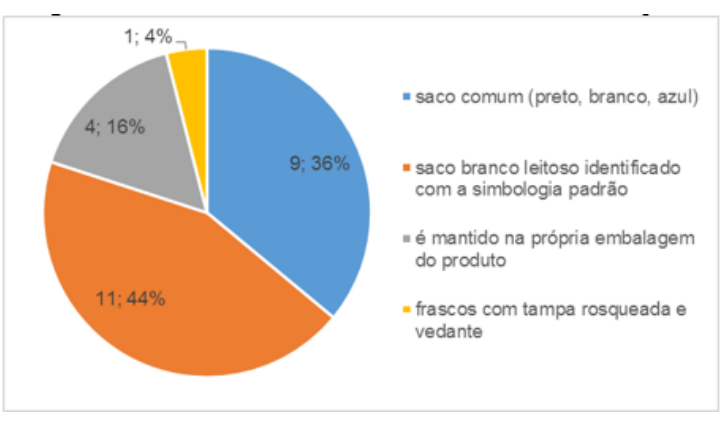

Os resíduos do Grupo D são, em 64\% dos estabelecimentos, acondicionados corretamente em saco comum, $20 \%$ utilizam saco branco leitoso desnecessariamente e $16 \%$ utilizam frascos com tampa rosqueada e vedante, Figura 4 .

Com relação ao acondicionamento dos resíduos do Grupo E, observou-se que $80 \%$ dos estabelecimentos utilizam, corretamente, recipiente rígido (caixas de papelão), $12 \%$ não souberam responder, $4 \%$ mantém o resíduo dentro da própria embalagem do produto e $4 \%$ utiliza saco branco leitoso, Figura 5 
Figura 4: Distribuição dos estabelecimentos visitados por bairro (Fortaleza, 2016)

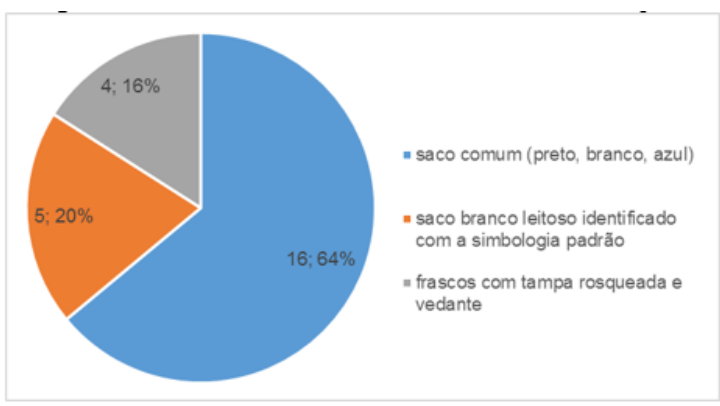

Figura 5: Distribuição dos estabelecimentos visitados por bairro (Fortaleza, 2016)

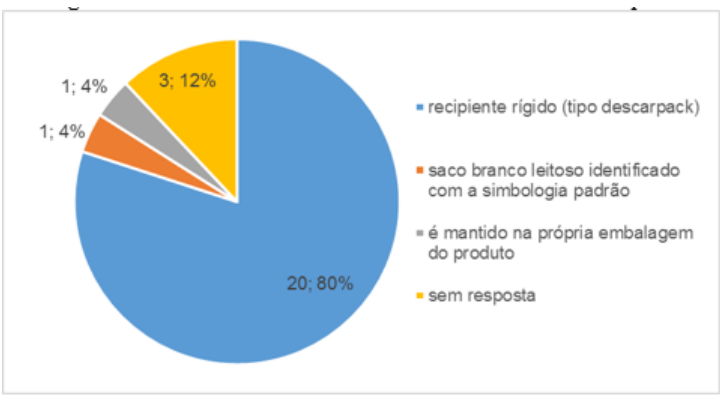

Conforme a Resolução da ANVISA no 306/2004 e do CONAMA $n^{\circ}$ 358/2005 acondicionar significa embalar os resíduos segregados, em sacos ou recipientes que evitem vazamentos e resistam às ações de punctura e ruptura. Por isso, a capacidade dos recipientes deve ser compatível com a geração diária de cada tipo de resíduo.

Nos casos onde o acondicionamento encontra-se inadequado várias situações são possíveis, especialmente a ruptura de embalagens, o derramamento dos resíduos e acidentes de trabalho. Essa situação contraria a Resolução da ANVISA n ${ }^{\circ} 306 / 2004$ e do CONAMA n ${ }^{\circ}$ $358 / 2005$.

Quando o acondicionamento é realizado de forma inadequada pode comprometer o resto do processo de gerenciamento dos resíduos Brasil (2006). Segundo Pilger e Schenato (2008) e Roeder-Ferrari, Filho e Ferrari (2008) há casos em que os resíduos infectantes e/ou químicos foram misturados com resíduos comuns, não só em sacos brancos como também em sacos pretos. Nas duas situações os autores citaram como causas: a segregação inadequada, a ineficácia da distribuição dos recipientes de acondicionamento e a falta de capacitação técnica do pessoal envolvido no manejo dos resíduos.

Moro (2010), em uma clínica veterinária, relata que o monitoramento do gerenciamento no local é realizado uma vez por mês, em dia aleatório, onde os sacos contendo os resíduos são abertos e o conteúdo é examinado quanto a sua devida segregação e identificação. Encontrando-se erros nessas etapas, deve ser identificado em qual ponto do processo o erro está acontecendo para ser corrigido.

A pesquisa de Nóbrega et al. (2000) mostrou que $62,5 \%$ dos estabelecimentos voltados à saúde animal separavam adequadamente os resíduos de serviços de saúde, mas só $43,75 \%$ acondicionavam de forma adequada. Já Pereira (2014) constatou que na etapa de acondicionamento apenas $38 \%$ dos estabelecimentos utilizavam sacos e recipientes adequados para embalar os resíduos.

A etapa posterior a de acondicionamento é a de identificação dos resíduos. Para a Resolução da ANVISA $n^{\circ} 306 / 2004$ essa etapa consiste no conjunto de medidas que permite o reconhecimento dos resíduos contidos nos sacos e recipientes, fornecendo informações ao correto manejo.

Na pesquisa realizada, observou-se que $80 \%$ dos estabelecimentos adotam medidas e estratégias (símbolos, frases, números) para identificação dos resíduos do Grupo E, 56\% identificam o Grupo A, 48\% (Grupo B) e $40 \%$ identificam os resíduos do Grupo D (Tabela 5).

Tabela 5: Estabelecimentos que realizam identificação dos resíduos

\begin{tabular}{l|l|l|l|l}
\hline \multirow{2}{*}{ OPÇÕES } & \multicolumn{4}{|c}{ GRUPO } \\
\cline { 2 - 5 } & $\mathrm{A}$ & $\mathrm{B}$ & $\mathrm{D}$ & $\mathrm{E}$ \\
\cline { 2 - 5 } & 56 & 48 & 40 & 80 \\
\hline Sim & 32 & 52 & 60 & 8 \\
\hline Não & 12 & - & - & 12 \\
\hline Sem resposta & \multicolumn{5}{|c}{100} \\
\hline TOTAL & \multicolumn{5}{|c}{ \% estab. } \\
\hline Fonte: autores & $(2016)$
\end{tabular}

A Resolução da ANVISA no 306/2004 traz um conjunto de orientações para se proceder com a correta identificação dos resíduos, a saber:

- A identificação deve estar posta nos sacos de acondicionamento, nos recipientes de coleta interna e externa, nos recipientes de transporte interno e externo, e nos locais de armazenamento, em local de fácil visualização, de forma indelével, utilizandose símbolos, cores e frases, atendendo aos parâmetros referenciados na norma NBR 7.500 da ABNT, além de outras exigências relacionadas à identificação de conteúdo e ao risco específico de cada grupo de resíduos. 
ANÁLISE MECÂNICA DE UM SOLO ARENO-ARGILOSO ESTABILIZADO COM CAL PARA FINS DE PAVIMENTAÇÃO

- A identificação dos sacos de armazenamento e dos recipientes de transporte poderá ser feita por adesivos, desde que seja garantida a resistência destes aos processos normais de manuseio dos sacos e recipientes.

- O Grupo A é identificado pelo símbolo de substância infectante constante na NBR-7500 da ABNT, com rótulos de fundo branco, desenho e contornos pretos.

- O Grupo B é identificado por meio do símbolo de risco associado, de acordo com a NBR-7500 da ABNT e com discriminação de substância química e frases de risco.

- O Grupo D é identificado pelo símbolo da reciclagem, com código de cores e suas correspondentes nomeações baseadas na Resolução Resolução... ().

- O Grupo E é identificado pelo símbolo de substância infectante constante na NBR-7500 da ABNT, com rótulos de fundo branco, desenho e contornos pretos, acrescido da inscrição de RESÍDUO PERFUROCORTANTE, indicando o risco que apresenta o resíduo ABNT (2004).

Por outro lado, a ausência de identificação (ou a identificação inadequada) prejudica o manejo dos resíduos e podem facilitar acidentes com trabalhadores e elevar dos custos de tratamento. Segundo Pilger e Schenato (2008) e Alves (2010) um dos fatores que contribui para segregação deficiente dos resíduos é o fato das áreas estarem desprovidas de recipientes adequados em tamanho, tipo e identificação.

Roeder-Ferrari, Filho e Ferrari (2008) observaram em um hospital veterinário que a única identificação dos resíduos era a existente na própria caixa de acondicionamento dos perfurocortantes (Grupo E) e nos sacos branco-leitosos (Grupo A), mas não haviam símbolos ou risco associados.

Em estabelecimentos veterinários de Mossoró-RN, Pereira (2014) observou que apenas 5\% realizavam a etapa de identificação dos resíduos.

Após a etapa de identificação, os resíduos devem sofrer transporte interno, que consiste no seu traslado dos pontos de geração até o local destinado ao armazenamento temporário ou armazenamento externo com a finalidade de apresentação para a coleta.

Nessa pesquisa foram encontrados cenários diferentes para cada grupo de resíduo, pois em alguns estabelecimentos a coleta interna é realizada sempre pela mesma pessoa, enquanto que em outros é realizada por qualquer pessoa (Tabela 6.

No primeiro caso, pode-se considerar que o risco de adoecimento de outros colaboradores e clientes "está
Tabela 6: Responsáveis pelo transporte interno dos resíduos

\begin{tabular}{l|l|l|l|l}
\hline \multirow{2}{*}{ OPÇÕES } & \multicolumn{4}{|c}{ GRUPO } \\
\cline { 2 - 5 } & $\mathrm{A}$ & $\mathrm{B}$ & $\mathrm{D}$ & $\mathrm{E}$ \\
\cline { 2 - 5 } & \multicolumn{4}{|c}{$\%$ de estab. } \\
\hline Sempre a mesma pessoa & 48 & 60 & 56 & 48 \\
\hline Qualquer pessoa & 40 & 40 & 44 & 40 \\
\hline Sem resposta & 12 & - & - & 12 \\
\hline TOTAL & \multicolumn{5}{|c}{100} \\
\hline Fonte: autores & $(2016)$
\end{tabular}

sob controle", desde que a pessoa tenha recebido orientação adequada sobre esse tipo de serviço. Caso contrário, o estabelecimento está, rotineiramente, pondo em risco a saúde desse(a) colaborador(a). No segundo caso, pode-se considerar um cenário de ampliação dos riscos, pois várias pessoas estão tendo acesso aos resíduos. Isso ocorre em até $44 \%$ dos estabelecimentos. Os resultados obtidos permitem ainda outra reflexão: alguns estabelecimentos utilizam apenas sacos plásticos no transporte interno dos resíduos e isso possibilita que trabalhadores ou clientes possam entrar em contato com esses resíduos, já que o correto seria transportá-los em recipiente de material rígido. Por outro lado, alguns estabelecimentos utilizam apenas os recipientes rígidos e isso poderá, no momento do recolhimento dos resíduos pela coleta externa, provocar danos aos trabalhadores.

Esse problema também foi percebido por Pereira (2014) e, na pesquisa de Roeder-Ferrari, Filho e Ferrari (2008), foi observado que a coleta interna era efetuada de forma manual, sendo os sacos retirados dos respectivos recipientes e levados até o local de armazenamento temporário.

As melhores orientações sobre o transporte interno dos resíduos podem ser encontradas na Resolução da ANVS (2004), a saber: o transporte interno de resíduos deve ser realizado atendendo roteiro previamente definido e em horários não coincidentes com a distribuição de roupas, alimentos e medicamentos, períodos de visita ou de maior fluxo de pessoas ou de atividades. Deve ser feito separadamente de acordo com o grupo de resíduos e em recipientes específicos. Os recipientes para transporte interno devem ser constituídos de material rígido, lavável, impermeável, provido de tampa articulada ao próprio corpo do equipamento, cantos e bordas arredondados, e serem identificados com o símbolo correspondente ao risco do resíduo neles contidos. Devem ser providos de rodas revestidas de material que reduza o ruído. Os recipientes com mais de 400 litros de capacidade devem possuir válvula de dreno no fundo. $\mathrm{O}$ uso de recipientes desprovidos de rodas deve observar os limites de carga permitidos para o transporte pelos trabalhadores, conforme normas reguladoras do Ministério do Trabalho e Emprego.

A etapa posterior a de transporte interno dos resíduos é a de tratamento. A Resolução da ANVISA n ${ }^{\circ}$ 
306/2004 define o tratamento como a aplicação de método, técnica ou processo que modifique as características dos riscos inerentes aos resíduos, reduzindo ou eliminando o risco de contaminação, de acidentes ocupacionais ou de dano ao meio ambiente. $\mathrm{O}$ tratamento térmico de resíduos quer seja nas próprias instalações do estabelecimento ou em outro local deve atender à Resolução CONAMA (2002).

Nessa pesquisa observou-se que $88 \%$ dos estabelecimentos adotam alguma medida de tratamento dos seus resíduos enquanto $12 \%$ não aplica nenhum método, técnica ou processo. Nos casos onde há tratamento interno, a Resolução da ANVISA no 306/2004 atribui ao praticante a responsabilidade sobre a garantia da eficácia dos equipamentos utilizados (ex. autoclave). No caso onde o tratamento é externo, os sistemas devem obedecer ao estabelecido na Resolução CONAMA (2002) (ex.: incineração).

Alves (2010) e Pereira (2014) revelaram resultados contrários aos dessa pesquisa. Os estabelecimentos pesquisados pelos autores não efetuavam nenhum método que modificasse as características dos resíduos para diminuir seus riscos de contaminação, com exceção de alguns tipos de resíduos do Grupo A. Já Nóbrega et al. (2000) informaram que apenas 6,25\% do total das clínicas e consultórios veterinários utilizavam a autoclave.

Após a etapa de tratamento (ou caso ela inexista) os resíduos devem ser armazenados em área externa. A Resolução da ANVS (2004) define como armazenamento externo a guarda dos recipientes de resíduos até a realização da etapa de coleta externa, em ambiente exclusivo com acesso facilitado para os veículos coletores.

Na pesquisa realizada, observou-se que 14 estabelecimentos $(56 \%)$ possuíam abrigo de resíduos (popular lixeira), 9 não possuía (36\%) e 2 não souberam responder $(8 \%)$. A ausência de abrigo pode acarretar vários problemas ambientais e de saúde pública, especialmente para os funcionários da coleta externa e populares. Além disso, a Lei Municipal $\mathrm{n}^{\circ} 10.340 / 2015$ prevê multa de $\mathrm{R} \$ 687,50$ para os estabelecimentos que não possuem abrigo adequado para armazenamento dos resíduos.

Na pesquisa desenvolvida por Alves (2010) também não foram encontrados abrigos externos para os resíduos, mas sim dois contêineres para a realização dessa etapa do manejo. Como ressaltaram Eliam I.S.V.; Barros (2004) os contêineres não devem substituir os abrigos, mas permanecer dentro destes.

Um aspecto verificado na pesquisa de Lippel (2003) também precisa ser considerado para ampliar o entendimento dessa questão: muitos estabelecimentos de pequeno porte não possuem espaço para construir um abrigo de resíduos. Em Pereira (2014) foi verificado outro cenário: $29 \%$ dos estabelecimentos que disseram possuir armazenamento externo utilizavam o local de forma compartilhada com outros serviços (guarda de produtos de limpeza ou até mesmo depósito) e nem sempre os abrigos possuíam pisos.

Nessa pesquisa, como anunciado anteriormente, foram encontrados 14 abrigos de resíduos, que em parte atendem as diretrizes municipais (Tabela 7), pois apresentam piso impermeável, ponto de luz e água e, acesso restrito. Por outro lado, a Instrução Normativa $n^{\circ}$ 08/08/2016 da Secretaria de Urbanismo e Meio Ambiente (SEUMA) exige sistema de combate a incêndio, sistema elétrico protegido contra fogo e explosão, altura mínima de $1,80 \mathrm{~m}$, cobertura e ventilação; quando se tratar de resíduos perigosos.

Tabela 7: Situação dos 14 abrigos de resíduos encontrados nos estabelecimentos.

\begin{tabular}{l|c} 
heightCONDIÇÕES & ESTAB. \\
\hline Tem piso impermeável & 14 \\
\hline Tem ponto de água & 14 \\
\hline Tem acesso restrito & 14 \\
\hline Tem ponto de luz & 11 \\
\hline Está superdimensionada & 6 \\
\hline Está subdimensionada & 8 \\
\hline
\end{tabular}

Fonte: Autores (2016).

Após a etapa de armazenamento, os resíduos devem sofrer coleta externa. A Resolução da ANVISA n ${ }^{\circ}$ 306/2004 define essa atividade como a remoção dos resíduos do abrigo de resíduos até a unidade de tratamento ou disposição final, utilizando-se técnicas que garantam a preservação das condições de acondicionamento e a integridade dos trabalhadores, da população e do meio ambiente, devendo estar de acordo com as orientações dos órgãos de limpeza urbana.

$\mathrm{Na}$ pesquisa realizada foram encontrados cenários diferentes para cada grupo de resíduo (Tabela 8), sendo majoritária a contratação de empresas especializadas para coleta dos resíduos dos grupos perigosos (A, B e E), portanto, atendendo as exigências na ANVISA, do CONAMA e da Prefeitura de Fortaleza. Observou-se

Tabela 8: Responsável pela coleta externa dos resíduos por grupo

\begin{tabular}{l|l|l|l|l}
\hline \multirow{2}{*}{ OPÇÕES } & \multicolumn{4}{|c}{ GRUPO } \\
\cline { 2 - 5 } & $\mathrm{A}$ & $\mathrm{B}$ & $\mathrm{D}$ & $\mathrm{E}$ \\
\cline { 2 - 5 } & \multicolumn{4}{|c}{$\%$ de estab. } \\
\hline Empresa contratada & 64 & 64 & 16 & 68 \\
\hline Coleta pública & 24 & 36 & 84 & 20 \\
\hline Sem resposta & 12 & - & - & 12 \\
\hline TOTAL & \multicolumn{5}{|c}{100} \\
\hline Fonte: autores & $(2016)$
\end{tabular}

ainda que alguns locais visitados estão colocando seus resíduos perigosos para coleta pública, o que não é permitido. Em Fortaleza-CE, a Lei $n^{0}$ 10.340/2015 disciplina o assunto e prevê que destinar resíduos perigosos sem tratamento prévio ou mesmo colocá-los para a coleta pública pode representar multa de até $\mathrm{R} \$ 17.187,50$. 
Além disso, cabe destacar que os resíduos coletados pelo sistema público vão direto para o aterramento sanitário, que não é adequado para resíduos de serviço de saúde. É verdade também que cresce a discussão sobre a real importância dos aterros sanitários, principalmente quando se observa os objetivos e princípios da Política Nacional de Resíduos Sólidos. Outro ponto a destacar na Tabela 9 , é que $84 \%$ dos geradores de resíduos do Grupo D (resíduos equiparados aos domiciliares) estão usufruindo da coleta pública, sendo que esse serviço só é permitido pela legislação municipal se o estabelecimento for enquadrado como pequeno gerador, ou seja, se produzir até 100 litros/dia de resíduos.

Conforme refletiu Pereira (2014), clínicas ou pets shops de pequeno porte tem grandes dificuldades em cumprir a legislação.

Todas as etapas até aqui discutidas, segundo a Resolução CONAMA n ${ }^{\circ}$ 358/2005, devem constar no Plano de Gerenciamento de Resíduos de Serviços de SaúdePGRSS. Nessa pesquisa, $68 \%$ dos estabelecimentos afirmaram possuir o PGRSS, sendo que $64 \%$ se negou a mostrar o documento.

Os estabelecimentos em funcionamento sem PGRSS estão ilegais, podem ser multados em até $\mathrm{R} \$ 2.062,50$, além de outras sanções. Pela Lei Municipal 10.340/2015 os geradores de resíduos sólidos caracterizados como resíduos da Classe I (perigosos) - qualquer que seja o seu volume - são obrigados a elaborar, implementar e operacionalizar Plano de Gerenciamento de Resíduos Sólidos e ficam obrigados a submeter o Plano à análise e à aprovação do órgão competente. Além disso, devem ainda manter cópia do PGRSS disponível para consulta sob solicitação da autoridade sanitária ou ambiental competente, dos funcionários e do público em geral.

Reis et al. (2013) trazem uma reflexão mais profunda sobre a questão do gerenciamento dos resíduos de serviços de saúde, ao tratar da formação dos profissionais que atuam nesse ramo. Conforme os autores, 69,4\% dos responsáveis técnicos pelos estabelecimentos veterinários visitados em Salvador (BA) desconhecem a Resolução da ANVISA pertinente, $72,2 \%$ desconhecem o PGRSS e $86,1 \%$ desconhecem os critérios de classificação dos resíduos de serviços de saúde, o que os impossibilita de proceder de forma correta.

No Brasil, a Lei Federal no $12.305 / 2010$ (Art. 24) prevê que o plano de gerenciamento de resíduos sólidos é parte integrante do processo de licenciamento ambiental do empreendimento ou atividade. Nessa pesquisa, $76 \%$ dos estabelecimentos visitados afirmaram possuir Licença Ambiental, sendo que $64 \%$ se negou a mostrar o documento.

\section{CONCLUSÕES}

Na condição de geradores de Resíduos de Serviços de Saúde (RSS), os estabelecimentos voltados à saúde ani- mal carecem de fiscalização sistemática e precisam receber maior atenção por parte dos pesquisadores, para que melhores condições de gerenciamento sejam executadas e novos conhecimentos possam ser gerados.

Em Fortaleza-CE é praticamente inexistente as pesquisas sobre o tema estudado nesse trabalho, o que de certo modo enaltece os resultados aqui obtidos, ou seja, consolidou-se uma base de dados primários que poderão servir para outras pesquisas e tomadas de decisão.

Diante dos resultados obtidos foi possível considerar que o objetivo desse trabalho foi alcançado à medida em que diagnosticou as atuais formas de manejo dos RSS em alguns estabelecimentos médicos veterinários de Fortaleza-CE. Por outro lado, a real situação do município só será possível com a ampliação dessa iniciativa e em parceria com poder público.

O trabalho permitiu concluir também que nenhum dos 25 estabelecimentos visitados cumpre por completo e corretamente todas as etapas do manejo dos RSS, mas isso não exclui a existência de boas práticas em alguns locais e em determinadas fases do gerenciamento.

Os maiores esforços dos responsáveis pelos estabelecimentos concentram-se nas etapas iniciais do sistema de gerenciamento de resíduos, especialmente na segregação e identificação, enquanto que a etapa mais frágil é a de armazenamento. Outras etapas põem em risco a saúde de todos os trabalhadores e clientes.

$\mathrm{O}$ aspecto mais curioso de todo o trabalho foi perceber que os entrevistados não têm interesse em mostrar os documentos (PGRSS e Licença Ambiental) que afirmaram possuir, enquanto que o esperado é que o empreendedor tenha orgulho em provar, a quem possa interessar, que seu estabelecimento funciona dentro da lei.

O desenvolvimento de ações, projetos e programas de educação ambiental direcionados a esse segmento pode, além de melhor orientar os profissionais e responsáveis pelos estabelecimentos veterinários, contribuirá para o alcance de um sistema de gerenciamento de RSS mais adequado.

A principal sugestão para um trabalho futuro seria ampliar a pesquisa de forma a permitir traçar um panorama geral da cidade de Fortaleza-CE e utilizar os dados alcançados para propor alternativas que estejam voltadas à não-geração e a redução desses resíduos, conforme Política Nacional de Resíduos Sólidos.

\section{REFERÊNCIAS}

ABNT. Identificação para o transporte terrestre, manuseio, movimentação e armazenamento de produtos. 2004. Associação Brasileira de normas técnicas. NBR 7500.

\section{AFONSO, T.; BERDASCO, L.; MEDEIROS}

T REJOWSKI, M. Mercado pet em ascensão - hotelaria 
para cães e gatos em são paulo. Revista Brasileira de Pesquisa em Turismo, dez. 2008.

ALVES, C. B. Produção e manejo de resíduos de serviço de saúde gerados em hospital veterinário. Dissertação (Mestrado) — Programa de Pós-Graduação em Ciência Animal, Universidade Federal de Goiás, 2010 .

ANVS, A. Resolução RDC no 306, de 07 de dezembro de 2004. Dispõe sobre o Regulamento Técnico para o gerenciamento de resíduos de serviços de saúde. DOU,. Brasília, 2004.

BRASIL. Manual de gerenciamento de resíduos de serviços de saúde. Brasília, 2006. Ministério da Saúde. Agência Nacional de Vigilância Sanitária.

REPÚBLICA FEDERATIVA DO. Lei n 12.305. Institui a Política Nacional de Resíduos Sólidos e dá outras providências. Brasília, 2010.

Lei n 10.340. Brasília: Congresso Nacional. DOU, 2015. Dispõe sobre a manipulação dos resíduos sólidos.

Lei n 16.032. Brasília: Congresso Nacional. DOU, 2016. Institui a Política Estadual de Resíduos Sólidos no âmbito do estado do Ceará.

CASTRO, N. R. P. S.; CASTRO, M. C. A. A.; RIBEIRO, M. L.; RISSATO, M. L.; OLIVEIRA, L. C. Resíduos de serviço de saúde gerados em unidade de saúde de pequeno porte no município de Jaú-SP. Revista Uniara, n. 20, 2007.

CFMV. Cuidados com a Destinação de pêlos e animais mortos. 2013. Conselho Federal de Medicina Veterinária-CFMV. Disponível em: <http: //www.cfmv.org.br/portal/destaque.php?cod=1155> Acesso em: 26 out. 2018.

CONAMA. CONSELHO NACIONAL DE MEIO AMBIENTE ,Resolução no 316. Dispõe sobre procedimentos e critérios para o funcionamento de sistemas de tratamento térmico de resíduos. DOU., Brasília, DF. 2002. Brasília, 2002.

\section{CONAMA. CONSELHO NACIONAL DE MEIO} AMBIENTE, Resolução no 358. Dispõe sobre o tratamento e a disposição final dos resíduos dos serviços de saúde e dá outras providências. DOU., Brasília, DF. [S.1.], 2005.

CUSSIOL, N. A. d. M. Manual de gerenciamento de resíduos de serviços de saúde. Belo Horizonte: FEAM, 2008. 88 p. Fundação Estadual do Meio Ambiente (FEAM).
ELIAM I.S.V.; BARROS, I. L. K. T. A. S. A. Resíduos biológicos em serviços de análise de diálise: discussão sobre o seu gerenciamento. Revista Eletrônica de Enfermagem, v. 6, n. 3, p. 378-384, 2004.

GARCIA, L. P.; ZANETTI-RAMOS, B. G.

Gerenciamento dos resíduos de serviços de saúde: uma questão de biossegurança. Cad. Saúde Pública, v. 20, n. 3, p. 744-752, 2004.

LIPPEL, M. Modelo de gerenciamento de resíduos sólidos de saúde para pequenos geradores: O Caso de Blumenau-SC. 120 p. Dissertação (Mestrado) - Universidade Federal de Santa Catarina,Centro Tecnológico, 2003.

MORO, C. G. Gestão de Resíduos de Saúde em uma Clínica Veterinária. Dissertação (Mestrado) Universidade Federal do Rio Grande Sul, Faculdade de Veterinária,, 2010.

NÓBREGA, C. C.; SILVA, J. S. d.; FLORES, N. J. P.; LIMA, J. D. d.; RUBERG, C. et al. Resíduos sólidos de serviços de saúde oriundos de clínicas odontológicas e clínicas veterinárias da cidade de joão pessoa/PB-Brasil: resultados preliminares. In: ABES. Congreso Interamericano de Ingeniería Sanitaria y Ambiental, 27. [S.1.], 2000. p. 1-8.

OPAS. Guia para o manejo interno de resíduos sólidos em estabelecimentos de saúde. Brasília: OPAS-Organização Pan-Americana de Saúde, 1997. Tradução de Carol Castillo Argüello.

PEREIRA, C. d. S. Diagnóstico do gerenciamento dos resíduos sólidos de serviços de saúde nos estabelecimentos veterinários de Mossoró-RN. Dissertação (Mestrado) — Universidade Federal Rural do Semi-Árido, Rio Grande do Norte, 2014.

PILGER, R. R.; SCHENATO, F. Classificação dos resíduos de serviços de saúde de um hospital veterinário. Engenharia Sanitária e Ambiental, SciELO Brasil, v. 13, n. 1, p. 23-28, 2008.

RAMOS, Y. S.; et al. Vulnerabilidade no manejo dos resíduos de serviços de saúde de João Pessoa (PB, Brasil). Ciência \& Saúde Coletiva, SciELO Public Health, v. 16, p. 3553-3560, 2011.

REIS, M. A.; RANGEL-S, M. L.; MATTOS, C. M. d.; FRANKE, C. R. Conhecimento, prática e percepção sobre o gerenciamento de resíduos de serviços de saúde em estabelecimentos médicos veterinários de salvador, bahia. Revista Brasileira de Saúde e Produção Animal, v. 14, n. 2, 2013.

Resolução no 275. Brasília,: Congresso Nacional. DOU. CONAMA - CONSELHO NACIONAL DE 
MEIO AMBIENTE. . Dispõe sobre o código de cores

para os diferentes tipos de resíduos.DF.

ROEDER-FERRARI, L. D.; FILHO, J. M. A.;

FERRARI, M. V. Produção e manejo de resíduos

sólidos de saúde no hospital veterinário da ufpr.

Archives of Veterinary Science, v. 13, p. 26-30, 2008. 